\title{
PERIODIC SOLUTIONS FOR PERIODIC SECOND-ORDER DIFFERENTIAL EQUATIONS WITH VARIABLE POTENTIALS
}

\author{
JAUME LLIBRE ${ }^{1}$ AND AMMAR MAKHLOUF ${ }^{2}$
}

\begin{abstract}
We provide sufficient conditions for the existence of periodic solutions of the second-order differential equation with variable potentials $-\left(p x^{\prime}\right)^{\prime}(t)-r(t) p(t) x^{\prime}(t)+q(t) x(t)=f(t, x(t))$, where the functions $p(t)>0, q(t), r(t)$ and $f(t, x)$ are $\mathcal{C}^{2}$ and $T$-periodic in the variable $t$.
\end{abstract}

\section{Introduction AND STATEMENT OF THE MAIN RESUlt}

We want to study the periodic solutions of the second-order differential equation with variable potentials given by

$$
-\left(p x^{\prime}\right)^{\prime}(t)-r(t) p(t) x^{\prime}(t)+q(t) x(t)=f(t, x(t))
$$

where the functions $p(t)>0, q(t), r(t)$ and $f(t, x)$ are $T$-periodic. Here the prime denotes derivative with respect to the time $t$.

The $T$-periodic differential equation (1) has been considered by several authors Thus Liu, Ge and Gui [6] (see also [2]) studied it with $r(t)=0$. Graef, Kong and Wang [5] give an extensive analysis when the functions $p(t), q(t)$ and $r(t)$ are constant. More recently, Anderson and Avery [3] also studied the periodic solutions of the differential equation (1) with $p(t)>0, q(t)>0$ and $r(t) \geq 0$.

Here we study the periodic solutions of the differential equation (1) with the unique basic assumption that the functions $p(t)>0, q(t), r(t)$ and $f(t, x)$ are $\mathcal{C}^{2}$ and $T$-periodic in the variable $t$.

2010 Mathematics Subject Classification. 37G15, 37C80, 37C30.

Key words and phrases. periodic orbit, third-order differential equation, quadratic system, averaging theory. 
Instead of working with the $T$-periodic second-order differential equation (1) we shall work with the following equivalent $T$-periodic differential system of first-order

$$
\begin{aligned}
& x^{\prime}=y, \\
& y^{\prime}=\frac{q(t)}{p(t)} x-\left(r(t)+\frac{p^{\prime}(t)}{p(t)}\right) y-\frac{f(t, x)}{p(t)} .
\end{aligned}
$$

Our results on the periodic solutions of the differential system (2) are summarized in the next theorem.

Theorem 1. We consider the differential system (2) where the functions $p(t)>0, q(t), r(t)$ and $f(t, x)$ are $\mathcal{C}^{2}$ and $T$-periodic in the variable $t$. Then the following statements hold.

(i) For every simple zero $x_{0}^{*}$ of the function

$$
x_{0} \int_{0}^{T} q(t) d t-\int_{0}^{T} f\left(t, x_{0}\right) d t
$$

system (2) has a periodic solution $(x(t), y(t))$ such that $(x(0), y(0))$ is close to $\left(x_{0}^{*}, 0\right)$.

(ii) If $g(t)=\int_{0}^{t}\left(r(s)+\frac{p^{\prime}(s)}{p(s)}\right) d s$ is a T-periodic function in the variable $t$, then for every simple zero $x_{0}^{*}$ of the function

$$
x_{0} \int_{0}^{T} \frac{e^{g(t)} q(t)}{p(t)} d t-\int_{0}^{T} \frac{e^{g(t)} f\left(t, x_{0}\right)}{p(t)} d t
$$

system (2) has a periodic solution $(x(t), y(t))$ such that $(x(0), y(0))$ is close to $\left(x_{0}^{*}, 0\right)$.

(iii) If $g(t)=\int_{0}^{t} q(s) d s$ is a T-periodic function in the variable $t$, then for every simple zero $\left(x_{0}^{*}, y_{0}^{*}\right)$ of the system

$$
\begin{aligned}
& x_{0} \int_{0}^{T} \frac{g(t)}{p(t)} d t+y_{0} \int_{0}^{T} \frac{p(0)}{p(t)} d t=0 \\
& x_{0} \int_{0}^{T} \frac{g(t) h(t)}{p(0)} d t+\int_{0}^{T} \frac{f\left(t, x_{0}\right)}{p(0)} d t+y_{0} \int_{0}^{T} h(t) d t=0,
\end{aligned}
$$

where $h(t)=\frac{g(t)}{p(t)}+r(t)$, system (2) has a periodic solution $(x(t), y(t))$ such that $(x(0), y(0))$ is close to $\left(x_{0}^{*}, y_{0}^{*}\right)$. 
PERIODIC SOLUTIONS OF SECOND-ORDER DIFFERENTIAL EQUATIONS 3

(iv) If $g(t, x)=\int_{0}^{t} f(s, x) d s$ is a T-periodic function in the variable $t$, then for every simple zero $\left(x_{0}^{*}, y_{0}^{*}\right)$ of the system

$$
\begin{aligned}
& \int_{0}^{T} \frac{g\left(t, x_{0}\right)}{p(t)} d t-y_{0} \int_{0}^{T} \frac{p(0)}{p(t)} d t=0, \\
& x_{0} \int_{0}^{T} \frac{q(t)}{p(0)} d t-\int_{0}^{T} \frac{g\left(t, x_{0}\right) h\left(t, x_{0}\right)}{p(0)} d t+y_{0} \int_{0}^{T} h\left(t, x_{0}\right) d t=0,
\end{aligned}
$$

where $h\left(t, x_{0}\right)=\frac{g_{x}\left(t, x_{0}\right)}{p(t)}-r(t)$, system (2) has a periodic solution $(x(t), y(t))$ such that $(x(0), y(0))$ is close to $\left(x_{0}^{*}, y_{0}^{*}\right)$.

(v) If $g(t)=\int_{0}^{t} \frac{1}{p(s)} d s$ is a T-periodic function in the variable $t$, then for every simple zero $\left(x_{0}^{*}, y_{0}^{*}\right)$ of the system

$$
\begin{gathered}
\int_{0}^{T} g(t) f\left(t, x_{0}+y_{0} p(0) g(t)\right) d t-x_{0} \int_{0}^{T} g(t) q(t) d t \\
+y_{0} p(0) \int_{0}^{T} g(t) h(t) d t=0, \\
x_{0} \int_{0}^{T} \frac{q(t)}{p(0)} d t-\int_{0}^{T} \frac{f\left(t, x_{0}+y_{0} P(0) g(t)\right)}{p(0)} d t-y_{0} \int_{0}^{T} h(t) d t=0,
\end{gathered}
$$

where $h(t)=r(t)-g(t) q(t)$, system (2) has a periodic solution $(x(t), y(t))$ such that $(x(0), y(0))$ is close to $\left(x_{0}^{*}, y_{0}^{*}\right)$.

(vi) If the functions $g(t)=\int_{0}^{t} \frac{p(s) r(s)+p^{\prime}(s)}{p(s)} d s$ and $h(t)=\int_{0}^{t} e^{-g(s)} d s$ are T-periodic in the variable $t$, then for every simple zero $\left(x_{0}^{*}, y_{0}^{*}\right)$ of the system

$$
\begin{gathered}
x_{0} \int_{0}^{T} \frac{h(t) q(t) e^{g(s)}}{p(t)} d t+y_{0} \int_{0}^{T} \frac{h^{2}(t) e^{g(s)} q(t)}{p(t)} d t- \\
\int_{0}^{T} \frac{h(t) e^{g(s)} f\left(t, x_{0}+h(t) y_{0}\right)}{p(t)} d t=0, \\
x_{0} \int_{0}^{T} \frac{q(t) e^{g(s)}}{p(t)} d t+y_{0} \int_{0}^{T} \frac{h(t) q(t) e^{g(s)}}{p(t)} d t- \\
\int_{0}^{T} \frac{e^{g(s)} f\left(t, x_{0}+y_{0} h(t)\right)}{p(t)} d t=0 .
\end{gathered}
$$


system (2) has a periodic solution $(x(t), y(t))$ such that $(x(0), y(0))$ is close to $\left(x_{0}^{*}, y_{0}^{*}\right)$.

Theorem 1 is proved in section 2. Its proof is based in the averaging theory and in different rescalings of the functions $p(t), q(t), r(t)$ and $f(t, x)$, and of the variables $x$ and $y$. In the appendix we recall the basic result of the averaging theory that we need for proving our results.

Finally in section 3 we provide examples of every one of the statements of Theorem 1.

\section{Proof of Theorem 1}

We do the following rescaling of the functions and variables which appear in the differential system (2)

$$
\begin{aligned}
x & =\varepsilon^{m_{1}} X, \\
y & =\varepsilon^{m_{2}} Y, \\
p(t) & =\varepsilon^{n_{1}} P(t), \\
q(t) & =\varepsilon^{n_{2}} Q(t), \\
r(t) & =\varepsilon^{n_{3}} R(t), \\
f(t, x) & =\varepsilon^{n_{4}} F\left(t, \varepsilon^{m_{1}} X\right),
\end{aligned}
$$

where $\varepsilon>0$ is a small parameter, and $m_{1}, m_{2}, n_{1}, n_{2}, n_{3}$ and $n_{4}$ are non-negative integers. Then the differential system (2) becomes

$$
\begin{aligned}
X^{\prime}= & \varepsilon^{m_{2}-m_{1}} Y \\
Y^{\prime}= & -\frac{P^{\prime}(t)}{P(t)} Y+\varepsilon^{m_{1}-m_{2}-n_{1}+n_{2}} \frac{Q(t)}{P(t)} X-\varepsilon^{n_{3}} R(t) Y \\
& -\varepsilon^{-m_{2}-n_{1}+n_{4}} \frac{F(t, X)}{P(t)} .
\end{aligned}
$$

We shall prove Theorem 1 statement by statement.

Proof of statement $(i)$ of Theorem 1. Taking $m_{1}=n_{1}=0, m_{2}=n_{3}=$ 1 and $n_{2}=n_{4}=2$ the differential system (4) writes

$$
X^{\prime}=\varepsilon Y,
$$

$$
Y^{\prime}=-\frac{P^{\prime}(t)}{P(t)} Y+\varepsilon\left(\frac{Q(t)}{P(t)} X-R(t) Y-\frac{F(t, X)}{P(t)}\right) .
$$


This system is in the normal form (15) for applying the averaging theory described in the appendix. More precisely, we have $\mathbf{x}=(X, Y)$,

$$
F_{0}(t, \mathbf{x})=\left(\begin{array}{c}
0 \\
-\frac{P^{\prime}(t)}{P(t)} Y
\end{array}\right), F_{1}(t, \mathbf{x})=\left(\begin{array}{c}
Y \\
\frac{Q(t)}{P(t)} X-R(t) Y-\frac{F(t, X)}{P(t)}
\end{array}\right),
$$

and $F_{2}(t, \mathbf{x})=0$.

Solving the differential system (16) we obtain the periodic solutions

$$
(X(t), Y(t))=\left(X_{0}, \frac{P(0)}{P(t)} Y_{0}\right)
$$

for all $\left(X_{0}, Y_{0}\right) \in \mathbb{R}^{2} \backslash\{(0,0)\}$. So all the solutions of the differential system (16) are $T$-periodic. Using the notation of the appendix we have that $\mathbf{z}=\left(X_{0}, Y_{0}\right)$. Now solving the variational differential equation (17) we obtain the fundamental matrix

$$
M_{\mathbf{z}}(t)=\left(\begin{array}{cc}
1 & 0 \\
0 & \frac{P(0)}{P(t)}
\end{array}\right) .
$$

We compute the function $\mathcal{F}(\mathbf{z})=\left(\mathcal{F}_{1}\left(X_{0}, Y_{0}\right), \mathcal{F}_{2}\left(X_{0}, Y_{0}\right)\right)$ defined in (18) and we get

$$
\begin{aligned}
& \mathcal{F}_{1}=Y_{0} \int_{0}^{T} \frac{P(0)}{P(t)} d t \\
& \mathcal{F}_{2}=\frac{1}{P(0)}\left(X_{0} \int_{0}^{T} Q(t) d t-Y_{0} P(0) \int_{0}^{T} R(t) d t-\int_{0}^{T} F\left(t, X_{0}\right) d t\right) .
\end{aligned}
$$

By Theorem 2 the differential system (10) has a periodic solution $(X(t, \varepsilon), Y(t, \varepsilon))$ such that

$$
(X(0, \varepsilon), Y(0, \varepsilon)) \rightarrow\left(X_{0}^{*}, 0\right) \quad \text { when } \varepsilon \rightarrow 0,
$$

for each simple zero $X_{0}^{*}$ of the function

$$
X_{0} \int_{0}^{T} Q(t) d t-\int_{0}^{T} F\left(t, X_{0}\right) d t .
$$

Going back to the differential system (2) through the rescaling (3) statement (i) follows. 
Proof of statement (ii) of Theorem 1. Taking $m_{1}=n_{1}=n_{3}=0, m_{2}=$ 1 and $n_{2}=n_{4}=2$ the differential system (4) writes

$$
X^{\prime}=\varepsilon Y \text {, }
$$

$$
Y^{\prime}=-\left(\frac{P^{\prime}(t)}{P(t)}+R(t)\right) Y+\varepsilon\left(\frac{Q(t)}{P(t)} X-\frac{F(t, X)}{P(t)}\right) .
$$

This system is in the normal form (15) for applying the averaging theory. We have $\mathbf{x}=(X, Y)$,

$$
F_{0}(t, \mathbf{x})=\left(\begin{array}{c}
0 \\
-\left(\frac{P^{\prime}(t)}{P(t)}+R(t)\right) Y
\end{array}\right), F_{1}(t, \mathbf{x})=\left(\begin{array}{c}
Y \\
\frac{Q(t)}{P(t)} X-\frac{F(t, X)}{P(t)}
\end{array}\right),
$$

and $F_{2}(t, \mathbf{x})=0$.

Solving the differential system (16) we obtain the periodic solutions

$$
(X(t), Y(t))=\left(X_{0}, \exp (-g(t)) Y_{0}\right),
$$

for all $\left(X_{0}, Y_{0}\right) \in \mathbb{R}^{2} \backslash\{(0,0)\}$, where we assume that the function $g(t)=\int_{0}^{t} \frac{P(s) R(s)+P^{\prime}(s)}{P(s)} d s$ is $T$-periodic. So all the solutions of the differential system (16) are $T$-periodic. Now we have that $\mathbf{z}=\left(X_{0}, Y_{0}\right)$, and solving the variational differential equation (17) we obtain the fundamental matrix

$$
M_{\mathbf{z}}(t)=\left(\begin{array}{cc}
1 & 0 \\
0 & e^{-g(t)}
\end{array}\right) .
$$

We compute the function $\mathcal{F}(\mathbf{z})=\left(\mathcal{F}_{1}\left(X_{0}, Y_{0}\right), \mathcal{F}_{2}\left(X_{0}, Y_{0}\right)\right)$ defined in (18) and we get

$$
\begin{aligned}
& \mathcal{F}_{1}=Y_{0} \int_{0}^{T} e^{-g(t)} d t \\
& \mathcal{F}_{2}=X_{0} \int_{0}^{T} \frac{e^{g(t)} Q(t)}{P(t)} d t-\int_{0}^{T} \frac{e^{g(t)} F\left(t, X_{0}\right)}{P(t)} d t .
\end{aligned}
$$

By Theorem 2 the differential system (10) has a periodic solution $(X(t, \varepsilon), Y(t, \varepsilon))$ such that

$$
(X(0, \varepsilon), Y(0, \varepsilon)) \rightarrow\left(X_{0}^{*}, 0\right) \quad \text { when } \varepsilon \rightarrow 0,
$$

for each simple zero $X_{0}^{*}$ of the function

$$
X_{0} \int_{0}^{T} \frac{e^{g(t)} Q(t)}{P(t)} d t-\int_{0}^{T} \frac{e^{g(t)} F\left(t, X_{0}\right)}{P(t)} d t
$$


Going back to the differential system (2) through the rescaling (3) statement (ii) follows.

Proof of statement (iii) of Theorem 1. Taking $m_{1}=n_{1}=0, m_{2}=$ $n_{2}=n_{3}=1$ and $n_{4}=2$ the differential system (4) writes

$$
\begin{aligned}
X^{\prime} & =\varepsilon Y, \\
Y^{\prime} & =\frac{Q(t)}{P(t)} X-\frac{P^{\prime}(t)}{P(t)} Y-\varepsilon\left(R(t) Y+\frac{F(t, X)}{P(t)}\right) .
\end{aligned}
$$

This system is in the normal form (15) for applying the averaging theory. Thus we have $\mathbf{x}=(X, Y)$,

$$
F_{0}(t, \mathbf{x})=\left(\begin{array}{c}
0 \\
\frac{Q(t)}{P(t)} X-\frac{P^{\prime}(t)}{P(t)} Y
\end{array}\right), F_{1}(t, \mathbf{x})=\left(\begin{array}{c}
Y \\
-R(t) Y-\frac{F(t, X)}{P(t)}
\end{array}\right),
$$

and $F_{2}(t, \mathbf{x})=0$.

Solving the differential system (16) we obtain the periodic solutions

$$
(X(t), Y(t))=\left(X_{0}, \frac{X_{0} \int_{0}^{t} Q(s) d s+Y_{0} P(0)}{P(t)}\right),
$$

for all $\left(X_{0}, Y_{0}\right) \in \mathbb{R}^{2} \backslash\{(0,0)\}$ where we suppose that the function $\int_{0}^{t} Q(s) d s$ is $T$-periodic. So all the solutions of the differential system (16) are $T$-periodic. Again we have that $\mathbf{z}=\left(X_{0}, Y_{0}\right)$, and solving the variational differential equation (17) we obtain the fundamental matrix

$$
M_{\mathbf{z}}(t)=\left(\begin{array}{cc}
1 & 0 \\
\frac{g(t)}{P(t)} & \frac{P(0)}{P(t)}
\end{array}\right) .
$$

where $g(t)=\int_{0}^{t} Q(s) d s$. We compute the function $\mathcal{F}(\mathbf{z})=\left(\mathcal{F}_{1}\left(X_{0}, Y_{0}\right)\right.$, $\left.\mathcal{F}_{2}\left(X_{0}, Y_{0}\right)\right)$ defined in (18) and we get

$$
\begin{aligned}
& \mathcal{F}_{1}=X_{0} \int_{0}^{T} \frac{g(t)}{P(t)} d t+Y_{0} \int_{0}^{T} \frac{P(0)}{P(t)} d t \\
& \mathcal{F}_{2}=X_{0} \int_{0}^{T} \frac{g(t) h(t)}{P(0)} d t+\int_{0}^{T} \frac{F\left(t, X_{0}\right)}{P(0)} d t+Y_{0} \int_{0}^{T} h(t) d t,
\end{aligned}
$$


where $h(t)=\frac{g(t)}{P(t)}+R(t)$. By Theorem 2 the differential system (10) has a periodic solution $(X(t, \varepsilon), Y(t, \varepsilon))$ such that

$$
(X(0, \varepsilon), Y(0, \varepsilon)) \rightarrow\left(X_{0}^{*}, Y_{0}^{*}\right) \quad \text { when } \varepsilon \rightarrow 0,
$$

for each zero $\left(X_{0}^{*}, Y_{0}^{*}\right)$ of the system,

$$
\mathcal{F}_{1}=0, \mathcal{F}_{2}=0
$$

whose Jacobian is different from zero. Going back to the differential system (2) through the rescaling (3) statement (iii) follows.

Proof of statement (iv) of Theorem 1. Taking $m_{1}=n_{1}=0, m_{2}=$ $n_{3}=n_{4}=1$ and $n_{2}=2$ the differential system (4) writes

$$
\begin{aligned}
& X^{\prime}=\varepsilon Y, \\
& Y^{\prime}=-\frac{P^{\prime}(t)}{P(t)} Y-\frac{F(t, X)}{P(t)}+\varepsilon\left(\frac{Q(t)}{P(t)} X-R(t) Y\right) .
\end{aligned}
$$

This system is in the normal form (15) for applying the averaging theory. Hence we have $\mathbf{x}=(X, Y)$,

$$
F_{0}(t, \mathbf{x})=\left(\begin{array}{c}
0 \\
-\frac{P^{\prime}(t)}{P(t)} Y-\frac{F(t, X)}{P(t)}
\end{array}\right), F_{1}(t, \mathbf{x})=\left(\begin{array}{c}
Y \\
\frac{Q(t)}{P(t)} X-R(t) Y
\end{array}\right),
$$

and $F_{2}(t, \mathbf{x})=0$.

Solving the differential system (16) we obtain the periodic solutions

$$
(X(t), Y(t))=\left(X_{0}, \frac{P(0)}{P(t)} Y_{0}-\frac{G\left(t, X_{0}\right)}{P(t)}\right),
$$

for all $\left(X_{0}, Y_{0}\right) \in \mathbb{R}^{2} \backslash\{(0,0)\}$ if $G\left(t, X_{0}\right)=\int_{0}^{t} F\left(s, X_{0}\right) d s$ is a Tperiodic function. So all the solutions of the differential system (16) are $T$-periodic. Therefore we have that $\mathbf{z}=\left(X_{0}, Y_{0}\right)$, and solving the variational differential equation (17) we obtain the fundamental matrix

$$
M_{\mathbf{z}}(t)=\left(\begin{array}{cc}
1 & 0 \\
-\frac{G_{x}\left(t, X_{0}\right)}{P(t)} & \frac{P(0)}{P(t)}
\end{array}\right) .
$$


We compute the function $\mathcal{F}(\mathbf{z})=\left(\mathcal{F}_{1}\left(X_{0}, Y_{0}\right), \mathcal{F}_{2}\left(X_{0}, Y_{0}\right)\right)$ defined in (18) and we get

$$
\begin{aligned}
& \mathcal{F}_{1}=Y_{0} \int_{0}^{T} \frac{P(0)}{P(t)} d t-\int_{0}^{T} \frac{G\left(t, X_{0}\right)}{P(t)} d t \\
& \mathcal{F}_{2}=X_{0} \int_{0}^{T} \frac{Q(t)}{P(0)} d t-\int_{0}^{T} \frac{G\left(t, X_{0}\right) H\left(t, X_{0}\right)}{P(0)} d t+Y_{0} \int_{0}^{T} H\left(t, X_{0}\right) d t,
\end{aligned}
$$

where $H\left(t, X_{0}\right)=\frac{G_{x}\left(t, X_{0}\right)}{P(t)}-R(t)$, By Theorem 2 the differential system (10) has a periodic solution $(X(t, \varepsilon), Y(t, \varepsilon))$ such that

$$
(X(0, \varepsilon), Y(0, \varepsilon)) \rightarrow\left(X_{0}^{*}, Y_{0}^{*}\right) \quad \text { when } \varepsilon \rightarrow 0,
$$

for each zero $\left(X_{0}^{*}, Y_{0}^{*}\right)$ of the system,

$$
\mathcal{F}_{1}=0, \mathcal{F}_{2}=0
$$

whose Jacobian is not zero. Going back to the differential system (2) through the rescaling (3) statement (iv) is proved.

Proof of statement $(v)$ of Theorem 1. Taking $m_{1}=m_{2}=n_{1}=0$ and $n_{2}=n_{3}=n_{4}=1$ the differential system (4) writes

$$
X^{\prime}=Y,
$$

$$
Y^{\prime}=-\frac{P^{\prime}(t)}{P(t)} Y+\varepsilon\left(\frac{Q(t)}{P(t)} X-R(t) Y-\frac{F(t, X)}{P(t)}\right) .
$$

This system is in the normal form (15) for applying the averaging theory. More precisely, we have $\mathbf{x}=(X, Y)$,

$$
F_{0}(t, \mathbf{x})=\left(\begin{array}{c}
Y \\
-\frac{P^{\prime}(t)}{P(t)} Y
\end{array}\right), F_{1}(t, \mathbf{x})=\left(\begin{array}{c}
0 \\
\frac{Q(t)}{P(t)} X-R(t) Y-\frac{F(t, X)}{P(t)}
\end{array}\right),
$$

and $F_{2}(t, \mathbf{x})=0$.

Solving the differential system (16) we obtain the periodic solutions

$$
(X(t), Y(t))=\left(X_{0}+Y_{0} P(0) G(t), \frac{P(0)}{P(t)} Y_{0}\right),
$$

for all $\left(X_{0}, Y_{0}\right) \in \mathbb{R}^{2} \backslash\{(0,0)\}$, where we assume that the function $G(t)=\int_{0}^{t} \frac{1}{P(s)} d s$ is T-periodic. So all the solutions of the differential system (16) are $T$-periodic. Now $\mathbf{z}=\left(X_{0}, Y_{0}\right)$ and solving the 
variational differential equation (17) we obtain the fundamental matrix

$$
M_{\mathbf{z}}(t)=\left(\begin{array}{cc}
1 & P(0) G(t) \\
0 & \frac{P(0)}{P(t)}
\end{array}\right)
$$

We compute the function $\mathcal{F}(\mathbf{z})=\left(\mathcal{F}_{1}\left(X_{0}, Y_{0}\right), \mathcal{F}_{2}\left(X_{0}, Y_{0}\right)\right)$ defined in (18) and we get

$$
\begin{aligned}
\mathcal{F}_{1}= & \int_{0}^{T} G(t) F\left(t, X_{0}+Y_{0} P(0) G(t)\right) d t-X_{0} \int_{0}^{T} G(t) Q(t) d t \\
& +Y_{0} P(0) \int_{0}^{T} G(t) H(t) d t \\
\mathcal{F}_{2}= & X_{0} \int_{0}^{T} \frac{Q(t)}{P(0)} d t-\int_{0}^{T} \frac{F\left(t, X_{0}+Y_{0} P(0) G(t)\right)}{P(0)} d t-Y_{0} \int_{0}^{T} H(t) d t,
\end{aligned}
$$

where $H(t)=R(t)-G(t) Q(t)$.

By Theorem 2 the differential system (10) has a periodic solution $(X(t, \varepsilon), Y(t, \varepsilon))$ such that

$$
(X(0, \varepsilon), Y(0, \varepsilon)) \rightarrow\left(X_{0}^{*}, Y_{0}^{*}\right) \quad \text { when } \varepsilon \rightarrow 0,
$$

for each zero $\left(X_{0}^{*}, Y_{0}^{*}\right)$ of the system $\mathcal{F}_{1}=0, \mathcal{F}_{2}=0$, whose Jacobian is not zero. Going back to the differential system (2) through the rescaling (3) statement (v) is proved.

Proof of statement (vi) of Theorem 1. Taking $m_{1}=m_{2}=n_{1}=n_{3}=$ 0 and $n_{2}=n_{4}=1$ the differential system (4) writes

$$
\begin{aligned}
& X^{\prime}=Y, \\
& Y^{\prime}=-\left(\frac{P^{\prime}(t)}{P(t)}+R(t)\right) Y+\varepsilon\left(\frac{Q(t)}{P(t)} X-\frac{F(t, X)}{P(t)}\right) .
\end{aligned}
$$

This system is in the normal form (15) for applying the averaging theory. More precisely, we have $\mathbf{x}=(X, Y)$,

$$
F_{0}(t, \mathbf{x})=\left(\begin{array}{c}
Y \\
-\left(\frac{P^{\prime}(t)}{P(t)}+R(t)\right) Y
\end{array}\right), F_{1}(t, \mathbf{x})=\left(\begin{array}{c}
0 \\
\frac{Q(t)}{P(t)} X-\frac{F(t, X)}{P(t)}
\end{array}\right),
$$

and $F_{2}(t, \mathbf{x})=0$.

Solving the differential system (16) we obtain the periodic solutions

$$
(X(t), Y(t))=\left(X_{0}+H(t) Y_{0}, e^{-G(t)} Y_{0}\right),
$$


for all $\left(X_{0}, Y_{0}\right) \in \mathbb{R}^{2} \backslash\{(0,0)\}=g(s)$, where we have assumed that the functions $G(t)=\int_{0}^{t} \frac{P(s) R(s)+P^{\prime}(s)}{P(s)} d s$ and $H(t)=\int_{0}^{t} e^{-G(u)} d u=$ $h(t)$ are $T$-periodic. So all the solutions (11) of the differential system (16) are $T$-periodic. Now putting $\mathbf{z}=\left(X_{0}, Y_{0}\right)$ and solving the variational differential equation (17) we obtain the fundamental matrix

$$
M_{\mathbf{z}}(t)=\left(\begin{array}{cc}
1 & H(t) \\
0 & e^{-G(t)}
\end{array}\right) .
$$

We compute the function $\mathcal{F}(\mathbf{z})=\left(\mathcal{F}_{1}\left(X_{0}, Y_{0}\right), \mathcal{F}_{2}\left(X_{0}, Y_{0}\right)\right)$ defined in (18) and we get

$$
\begin{aligned}
\mathcal{F}_{1}= & X_{0} \int_{0}^{T} \frac{H(t) Q(t) e^{G(t)}}{P(t)} d t+Y_{0} \int_{0}^{T} \frac{H^{2}(t) Q(t) e^{G(t)}}{P(t)} d t- \\
& \int_{0}^{T} \frac{H(t) e^{G(t)} F\left(t, X_{0}+H(t) Y_{0}\right)}{P(t)} d t \\
\mathcal{F}_{2}= & X_{0} \int_{0}^{T} \frac{Q(t) e^{G(t)}}{P(t)} d t+Y_{0} \int_{0}^{T} \frac{H(t) Q(t) e^{G(t)}}{P(t)} d t- \\
& \int_{0}^{T} \frac{e^{G(t)} F\left(t, X_{0}+H(t) Y_{0}\right)}{P(t)} d t .
\end{aligned}
$$

By Theorem 2 the differential system (10) has a periodic solution $(X(t, \varepsilon), Y(t, \varepsilon))$ such that

$$
(X(0, \varepsilon), Y(0, \varepsilon)) \rightarrow\left(X_{0}^{*}, Y_{0}^{*}\right) \quad \text { when } \varepsilon \rightarrow 0,
$$

for each zero $\left(X_{0}^{*}, Y_{0}^{*}\right)$ of the system $\mathcal{F}_{1}=0, \mathcal{F}_{2}=0$, whose Jacobian is not zero. Going back to the differential system (2) through the rescaling (3) statement (vi) is proved.

\section{EXAMPLES}

In this section we provide examples of each one of the statements of Theorem 1.

Example (i): Consider the differential system (2) with

$$
p(t)=2+\cos t, q(t)=a \neq 0, r(t)=1+\cos t, f(t, x)=\sin ^{2}(t+x) .
$$

All these functions are $2 \pi$-periodic in the variable $t$. Then applying statement (i) of Theorem 1 we have that the function

$$
x_{0} \int_{0}^{2 \pi} q(t) d t-\int_{0}^{2 \pi} f\left(t, x_{0}\right) d t=\pi\left(2 a x_{0}-1\right),
$$


has a unique simple zero $x_{0}^{*}=1 /(2 a)$. Therefore, the differential system (2) has a periodic solution $(x(t), y(t))$ such that $(x(0), y(0))$ is near $(1 /(2 a), 0)$. In fact, numerically we can find the periodic orbit for $a=1$ very near to $(0.55,0)$.

Example (ii): Consider the differential system (2) with

$$
p(t)=a>0, q(t)=b>0, r(t)=\cos t, f(t, x)=x^{3} \sin t .
$$

These functions are $2 \pi$-periodic in the variable $t$. We have that the function

$$
g(t)=\int_{0}^{t}\left(r(s)+\frac{p^{\prime}(s)}{p(s)}\right) d s=\sin t
$$

so it is also $2 \pi$-periodic. Now applying statement (ii) of Theorem 1 we have that the function

$$
x_{0} \int_{0}^{2 \pi} \frac{e^{g(t)} q(t)}{p(t)} d t-\int_{0}^{2 \pi} \frac{e^{g(t)} f\left(t, x_{0}\right)}{p(t)} d t=\frac{2 \pi}{a} x_{0}\left(b I_{0}(1)-x_{0}^{2} I_{1}(1)\right),
$$

where $I_{n}(z)$ is the modified Bessel function of the first kind, for more details see [1]. This function has three simple zeros $x_{0}^{*}$, namely

$$
0, \pm \sqrt{\frac{b I_{0}(1)}{I_{1}(1)}}
$$

where $I_{0}(1) / I_{1}(1)=2.24019 \ldots$ Therefore, the differential system $(2)$ has three periodic solution $(x(t), y(t))$ such that $(x(0), y(0))$ are near $\left(x_{0}^{*}, 0\right)$.

Example (iii): Consider the differential system (2) with

$$
p(t)=1, q(t)=\sin t, r(t)=1+\cos t, f(t, x)=x^{2}-1 .
$$

These functions are $2 \pi$-periodic in the variable $t$. We have that the function

$$
g(t)=\int_{0}^{t} q(s) d s=1-\cos t
$$

so it is also $2 \pi$-periodic. Now applying statement (iii) of Theorem 1 we obtain the system

$$
\begin{aligned}
\mathcal{F}_{1}\left(x_{0}, y_{0}\right) & =x_{0} \int_{0}^{2 \pi} \frac{g(t)}{p(t)} d t+y_{0} \int_{0}^{2 \pi} \frac{p(0)}{p(t)} d t=2 \pi\left(x_{0}+y_{0}\right)=0, \\
\mathcal{F}_{2}\left(x_{0}, y_{0}\right) & =x_{0} \int_{0}^{2 \pi} \frac{g(t) h(t)}{p(0)} d t+\int_{0}^{2 \pi} \frac{f\left(t, x_{0}\right)}{p(0)} d t+y_{0} \int_{0}^{2 \pi} h(t) d t \\
& =2 \pi\left(x_{0}^{2}+2\left(x_{0}+y_{0}\right)-1\right)=0 .
\end{aligned}
$$

This system has the two solutions $(1,-1)$ and $(-1,1)$. Since the Jacobian (19) is not zero in both solutions, the differential system (2) has 
two periodic solution $(x(t), y(t))$ such that $(x(0), y(0))$ are near these two points.

Example (iv): Consider the differential system (2) with

$$
p(t)=1, q(t)=\cos t, r(t)=\sin t, f(t, x)=x^{2} \cos t .
$$

These functions are $2 \pi$-periodic in the variable $t$. This system can be written as

$$
\begin{aligned}
& x^{\prime}=y, \\
& y^{\prime}=x \cos t-y \sin t-x^{2} \cos t .
\end{aligned}
$$

The function

$$
g(t, x)=\int_{0}^{t} f(s, x) d s=x^{2} \sin t,
$$

is $2 \pi$-periodic. Now applying statement (iv) of Theorem 1 we obtain the system

$$
\begin{aligned}
& \int_{0}^{2 \pi} \frac{g\left(t, x_{0}\right)}{p(t)} d t-y_{0} \int_{0}^{2 \pi} \frac{p(0)}{p(t)} d t=-2 \pi y_{0}=0 \\
& x_{0} \int_{0}^{2 \pi} \frac{q(t)}{p(0)} d t-\int_{0}^{2 \pi} \frac{g\left(t, x_{0}\right) h\left(t, x_{0}\right)}{p(0)} d t+y_{0} \int_{0}^{2 \pi} h\left(t, x_{0}\right) d t= \\
& -\pi x_{0}^{2}\left(2 x_{0}-1\right)=0,
\end{aligned}
$$

where $h\left(t, x_{0}\right)=g_{x}\left(t, x_{0}\right) / p(t)-r(t)$. This system has the two solutions $(0,0)$ and $(1 / 2,0)$, the first is not good because corresponds to an equilibrium point. Since the Jacobian (19) is $\pi^{2}$ for the second solution, the differential system (2) has one periodic solution $(x(t), y(t))$ such that $(x(0), y(0))$ is near $(1 / 2,0)$.

Example (v): Consider the differential system (2) with

$$
\begin{aligned}
& p(t)=\frac{1}{a \sin ^{2} t+b \cos ^{2} t-(a+b) / 2}, \\
& q(t)=\cos ^{2} t \\
& r(t)=-2 \tan (2 t), \\
& f(t, x)=\left(2 x+\sin ^{2} t\right) \cos ^{2} t .
\end{aligned}
$$

These functions are $2 \pi$-periodic in the variable $t$ where they are defined. Then system (2) becomes

$$
\begin{aligned}
& x^{\prime}=y, \\
& y^{\prime}=\frac{1}{2}(a-b) \cos ^{2} t \cos (2 t)\left(\sin ^{2} t+x\right),
\end{aligned}
$$

and it is defined for all $t \in \mathbb{R}$. 
The function

$$
g(t)=\int_{0}^{t} \frac{1}{p(s)} d s=-\frac{1}{4}(a-b) \sin (2 t)
$$

is $2 \pi$-periodic. Now applying statement $(\mathrm{v})$ of Theorem 1 we obtain the system

$$
\begin{array}{r}
\int_{0}^{T} g(t) f\left(t, x_{0}+y_{0} p(0) g(t)\right) d t-x_{0} \int_{0}^{T} g(t) q(t) d t \\
+y_{0} p(0) \int_{0}^{T} g(t) h(t) d t=\frac{1}{16}(b-a) \pi y_{0}=0 \\
x_{0} \int_{0}^{T} \frac{q(t)}{p(0)} d t-\int_{0}^{T} \frac{f\left(t, x_{0}+y_{0} p(0) g(t)\right)}{p(0)} d t-y_{0} \int_{0}^{T} h(t) d t \\
=\frac{1}{8}(a-b) \pi\left(1+4 x_{0}\right)=0,
\end{array}
$$

where where $h(t)=r(t)-g(t) q(t)$. This system has the unique solution $\left(x_{0}, y_{0}\right)=(-1 / 4,0)$. Since the Jacobian (19), for this solution is $(a-$ $b)^{2} \pi^{2} / 32$ which is different from zero for $a \neq b$, the differential system (2) has one periodic solution $(x(t), y(t))$ such that $(x(0), y(0))$ is near this point.

Example (vi): Consider the differential system (2) with

$$
p(t)=1, \quad q(t)=\cos t, \quad r(t)=\tan t, \quad f(t, x)=x^{2} \cos t .
$$

These functions are $2 \pi$-periodic in the variable $t$. Then system (2) becomes

$$
\begin{aligned}
& x^{\prime}=y, \\
& y^{\prime}=\left(x-y-x^{2}\right) \cos t,
\end{aligned}
$$

and it is defined for all $t \in \mathbb{R}$.

The functions

$$
g(t)=\int_{0}^{t} \frac{p(s) r(s)+p^{\prime}(s)}{p(s)} d s=-\log |\cos t|, \text { and } h(t)=\int_{0}^{t} e^{-g(s)} d s=\sin t
$$


are $2 \pi$-periodic. Now applying statement (vi) of Theorem 1 we obtain the system

$$
\begin{gathered}
x_{0} \int_{0}^{2 \pi} \frac{h(t) q(t) e^{g(s)}}{p(t)} d t+y_{0} \int_{0}^{2 \pi} \frac{h^{2}(t) e^{g(s)} q(t)}{p(t)} d t- \\
\int_{0}^{2 \pi} \frac{h(t) e^{g(s)} f\left(t, x_{0}+h(t) y_{0}\right)}{p(t)} d t=-\pi\left(2 x_{0}-1\right) y_{0}=0, \\
x_{0} \int_{0}^{2 \pi} \frac{q(t) e^{g(s)}}{p(t)} d t+y_{0} \int_{0}^{2 \pi} \frac{h(t) q(t) e^{g(s)}}{p(t)} d t- \\
\int_{0}^{2 \pi} \frac{e^{g(s)} f\left(t, x_{0}+y_{0} h(t)\right)}{p(t)} d t=-\pi\left(2 x_{0}^{2}-2 x_{0}+y_{0}^{2}\right)=0 .
\end{gathered}
$$

This system has the four solutions $(0,0),(1,0)$ and $(1 / 2, \pm 1 / \sqrt{2})$. The first two do not provide periodic solutions, they correspond to equilibrium points. Since the Jacobian (19), for the last two solutions is $2 \pi^{2}$, the differential system (2) has two periodic solutions $(x(t), y(t))$ such that $(x(0), y(0))$ are near the points $(1 / 2, \pm 1 / \sqrt{2})$.

\section{APPENDIX: AVERAGING THEORY OF FIRST ORDER}

In this appendix we recall one of the basic results from the averaging theory that we need for proving the main results of this paper.

We consider the problem of the bifurcation of $T$-periodic solutions from differential systems of the form

$$
\mathbf{x}^{\prime}=F_{0}(t, \mathbf{x})+\varepsilon F_{1}(t, \mathbf{x})+\varepsilon^{2} F_{2}(t, \mathbf{x}, \varepsilon)
$$

with $\varepsilon=0$ to $\varepsilon \neq 0$ sufficiently small. Here the functions $F_{0}, F_{1}$ : $\mathbb{R} \times \Omega \rightarrow \mathbb{R}^{n}$ and $F_{2}: \mathbb{R} \times \Omega \times\left(-\varepsilon_{0}, \varepsilon_{0}\right) \rightarrow \mathbb{R}^{n}$ are $\mathcal{C}^{2}$ functions, Tperiodic in the first variable, and $\Omega$ is an open subset of $\mathbb{R}^{n}$. The main assumption is that the unperturbed system

$$
\mathbf{x}^{\prime}=F_{0}(t, \mathbf{x}),
$$

has a submanifold of dimension $n$ of periodic solutions. A solution of this problem is given using the averaging theory.

Let $\mathbf{x}(t, \mathbf{z}, \varepsilon)$ be the solution of the system (16) such that $\mathbf{x}(0, \mathbf{z}, \varepsilon)=$ $\mathbf{z}$. We write the linearization of the unperturbed system along the periodic solution $\mathbf{x}(t, \mathbf{z}, 0)$ as

$$
\mathbf{y}^{\prime}=D_{\mathbf{x}} F_{0}(t, \mathbf{x}(t, \mathbf{z}, 0)) \mathbf{y},
$$

where $\mathbf{y}$ is an $n \times n$ matrix. In what follows we denote by $M_{\mathbf{z}}(t)$ some fundamental matrix of the linear differential system (17). 
We assume that there exists an open set $V$ with $\mathrm{Cl}(V) \subset \Omega$ such that for each $\mathbf{z} \in \mathrm{Cl}(V), \mathbf{x}(t, \mathbf{z}, 0)$ is $T$-periodic. The set $\mathrm{Cl}(V)$ is isochronous for the system (15); i.e. it is a set formed only by periodic orbits, all of them having the same period. Then, an answer to the problem of the bifurcation of $T$-periodic solutions from the periodic solutions $\mathbf{x}(t, \mathbf{z}, 0)$ contained in $\mathrm{Cl}(V)$ is given in the following result.

Theorem 2 (Perturbations of an isochronous set). We assume that there exists an open and bounded set $V$ with $\mathrm{Cl}(V) \subset \Omega$ such that for each $\mathbf{z} \in \mathrm{Cl}(V)$, the solution $\mathbf{x}(t, \mathbf{z}, 0)$ is $T$-periodic, then we consider the function $\mathcal{F}: \mathrm{Cl}(V) \rightarrow \mathbb{R}^{n}$

$$
\mathcal{F}(\mathbf{z})=\int_{0}^{T} M_{\mathbf{z}}^{-1}(t) F_{1}(t, \mathbf{x}(t, \mathbf{z}, 0)) d t .
$$

If there exists $\alpha \in V$ with $\mathcal{F}(\alpha)=0$ and

$$
\operatorname{det}\left((d \mathcal{F} / d \mathbf{z})\left(\mathbf{z}^{*}\right)\right) \neq 0,
$$

then there exists a $T$-periodic solution $\mathbf{x}(t, \varepsilon)$ of system (15) such that when $\varepsilon \rightarrow 0$ we have that $\mathbf{x}(0, \varepsilon) \rightarrow \alpha$.

Theorem 2 goes back to Malkin [7] and Roseau [8], for a shorter and easier proof see [4].

\section{ACKNOWLEDGEMENTS}

The first author is partially supported by a MICINN/FEDER grant number MTM2009-03437, by an AGAUR grant number 2009SGR-410, by an ICREA Academia, two FP7+PEOPLE+2012+IRSES numbers 316338 and 318999, and FEDER-UNAB10-4E-378.

\section{REFERENCES}

[1] M. Abramowitz and I.A. Stegun, Modified Bessel Functions I and K, $\S 9.6$ in Handbook of Mathematical Functions with Formulas, Graphs, and Mathematical Tables, 9th printing. New York: Dover, pp. 374-377, 1972.

[2] D.R. Anderson, Multiple periodic solutions for a second-order problem on periodic time scales, Nonlinear Anal. 60(1) (2005), 101-115.

[3] D.R. Anderson And R.I. Avery, Existence of a periodic solution for continuous and discrete periodic second-order equations with variable potentials, J. Appl. Math. Comput. 37 (2011), 297-312.

[4] A. Buica, J. P. Françoise and J. Llibre, Periodic solutions of nonlinear periodic differential systems with a small parameter, Comm. Pure Appl. Anal. 6 (2006), 103-111.

[5] J.R. Graef, L. Kong And H. Wang, Existence, multiplicity, and dependence on a parameter for a periodic boundary value problem, J. Differ. Equ. 245 (2008), 1185-1197. 
[6] Y. LiU, W. GE And Z. Gui, Three positive periodic solutions of nonlinear differential equations with periodic coefficients, Anal. Appl. 3(2) (2005), 145155.

[7] I.G. Malkin, Some Problems of the theory of nonlinear oscillations, Gosudarstv. Izdat. Tehn-Teor. Lit. Moscow, 1956 (in Russian).

[8] M. Roseau, Vibrations non linéaires et théorie de la stabilité, Springer Tracts in Natural Philosophy, Vol. 8, Springer, New York, 1985.

${ }^{1}$ Departament de Matematiques, Universitat Autònoma de Barcelona, 08193 Bellaterra, Barcelona, Catalonia, Spain

E-mail address: jllibre@mat.uab.cat

2 Department of Mathematics, University of Annaba, Elhadjar 23 Annaba, Algeria

E-mail address: makhloufamar@yahoo.fr 